\title{
NEW ATOMIC DATA FOR Mg I LINES
}

\author{
D. HOANG-BINH \\ LAM, Observatoire de Paris, F-92195 Meudon Cédex, France
}

\begin{abstract}
Theoretical oscillator strengths are reported for transitions between excited terms of the singlet and triplet $S, P, D, F$ and $G$ manifolds of atomic magnesium. The principal quantum numbers of the jumping electron range from $n=4$ to 100 . The calculations are based on the Coulomb approximation. Low- $n$ results, for which other data are available previously, agree reasonably well with the sophisticated calculations of Moccia and Spizzo (1988). Data for ${ }^{1,3} F-1,3 G$ transitions may deviate from hydrogenic values by as much as $50 \%$.
\end{abstract}

Key words: atomic data - infrared: stars - Mg I

\section{Introduction}

Absorption oscillator strengths for transitions in $\mathrm{Mg}$ I have been calculated by many authors, e.g., Froese-Fisher (1975a,b), Victor et al. (1976), Moccia and Spizzo (1988, hereafter MS). In these works, the principal quantum numbers of the jumping electron are smaller than 10. Over the last decade, several lines of $\mathrm{Mg} \mathrm{I}$ have been discovered in the far-infrared spectrum of the sun (e.g., Brault and Noyes 1983; Chang and Noyes 1983; Jefferies 1991), and their study has become an important topic in solar physics. As the recent works of Hoang-Binh (1991) and Chang et al. (1991) have shown, investigations on the formation of these Rydberg lines require a large body of atomic data, in particular for principal quantum numbers $n \geq 10$. We report new calculations of multiplet oscillator strengths for transitions between excited terms of $\mathrm{Mg} \mathrm{I}$, for $n$ up to 100 . These data are required to compute radiative and collisional transition rates entering the statistical equilibrium equations, and are also relevant to the study of the line broadening (Hoang-Binh et al. 1987).

\section{Theory}

The multiplet absorption oscillator strength, for a transition between a lower term $i$ and an upper term $k$, is given by (Wiese et al. 1969):

$$
f(i, k)=\left(303.7 / g_{i} \lambda\right) S_{i, k}
$$

where $\lambda$ is the wavelength of the transition in $\AA, S_{i, k}$ is the multiplet strength in atomic units (a.u.), and $g_{i}$ is the statistical weight of the lower term. The strength of a transition $3 s n l^{2 S+1} L$ to $3 s n^{\prime} l^{2 S+1} L^{\prime}$, involving no equivalent electrons, is, for $l^{\prime}=l-1$ (Goldberg 1939),

$$
S(i, k)=(2 S+1)(2 L+1) l(2 l-1) \sigma^{2},
$$

where

$$
\begin{aligned}
\sigma^{2} & =a_{0}^{2} e^{2} /\left(4 l^{2}-1\right) R^{2}, \\
R & =\int_{0}^{\infty} P_{n, l}(r) P_{n^{\prime}, l^{\prime}}(r) d r,
\end{aligned}
$$


and $n, n^{\prime}$ and $l, l^{\prime}$ are the principal and azimuthal quantum numbers respectively of the jumping electron; $S$ is the total spin and $L, L^{\prime}$ the total azimuthal quantum numbers, $a_{0}$ is the Bohr radius ( 1 in a.u.), and $P_{n, l}(r) a n d P_{n^{\prime}, l^{\prime}}(r)$ are normalized radial eigenfunctions, in atomic units, of the jumping electron in the relevant configurations.

Thus, the calculation of $f(i, k)$ reduces to that of the integral $R$. For $n, n^{\prime} \geq 10$, it is justified to use the Coulomb approximation, for which (Hoang-Binh et al. 1979)

$$
P_{n, l}(r)=z^{1 / 2} K(\nu, l) W_{\nu, l+1 / 2}(2 \rho / \nu),
$$

where $z$ is the effective charge, $\nu$ is the effective quantum number, $\rho=z r$, and $W_{\nu, l+1 / 2}$ is the Whittaker function. The normalizing factor is (Seaton 1958)

$$
K(\nu, l)=\left[\zeta(\nu) \nu^{2} \Gamma(\nu+l+1) \Gamma(\nu-l)\right]^{-1 / 2} .
$$

Provided $\nu$ is not too small, $\zeta(\nu) \approx 1$; here we shall take $\zeta(\nu)=1$ and use the analytical formula given by Hoang-Binh et al. (1979) to calculate $R$. The quantum defects have been calculated, using either the term positions of Bashkin and Stoner (1975) (for $n, n^{\prime}$ up to $\approx 8$ ), or the extended Ritz formulae given by Risberg (1965) for larger values of $n, n^{\prime}$. For ${ }^{1} D$ terms, values for large $n$ have been obtained by extrapolation of Risberg's values; ${ }^{1,3} G$ terms are taken to be hydrogenic.

\section{Results and Discussion}

Calculations have been performed for transitions between the ${ }^{1,3} S,{ }^{1,3} P^{0},{ }^{1,3} D$, and ${ }^{1,3} G$ manifolds. The principal quantum numbers of the jumping electron range from 4 or 5 to 100 . Although we are mainly interested in the domain $n, n^{\prime} \geq 10$, where data are generally not available, lower terms have been considered for the sake of self-consistency and for comparison with other theoretical results. The full results will be published elsewhere. Here, we show only the trends of $f$-values for large $n$ and $\Delta n$. Let the principal quantum numbers of the upper and lower terms be denoted by $m$ and $n$ respectively; and $\Delta n=m-n$. Figure 1 displays an example of the typical behavior of the oscillator strength with respect to $\Delta n$ (for $n$ fixed), and indicates that interpolation or extrapolation are possible. An exception concerns the transitions $n p^{1} P-m d^{1} D$, for which the variation of $f$ as a function of $\Delta n$ is not monotonic for $n<10$. In general, it is found that $f \sim 1 /\left(\Delta n^{\gamma}\right)$, with $\gamma \approx 2.7$ in the limit of large $\Delta n$, while $f$ is approximately linear with respect to $n$ in the limit of large $n$.

To our knowledge, the most extensive body of theoretical data on $\mathrm{Mg}$ I has been given by MS, who considered only $n, n^{\prime} \leq 9$. The agreement of our results with these sophisticated calculations is excellent in some instances, e.g., for the transitions $n p^{1} P-m s^{1} S$ (see Table 1). This is somewhat surprising, and probably fortuitous, since we do not expect the Coulomb approximation to be very good for transitions involving low $n$ and strongly core-penetrating orbits. One the other hand, a few large discrepancies may be also noted (e.g., $\left.n p^{1} P^{0}-m d^{1} D, \Delta n \geq 1\right)$. On the whole, most of our low- $n$ results agree reasonably well with those of MS. Our high- $n$ results should be more reliable, since the radial wave function is rather well represented by the Whittaker function at large radial distances. For Mg I, Wiese 


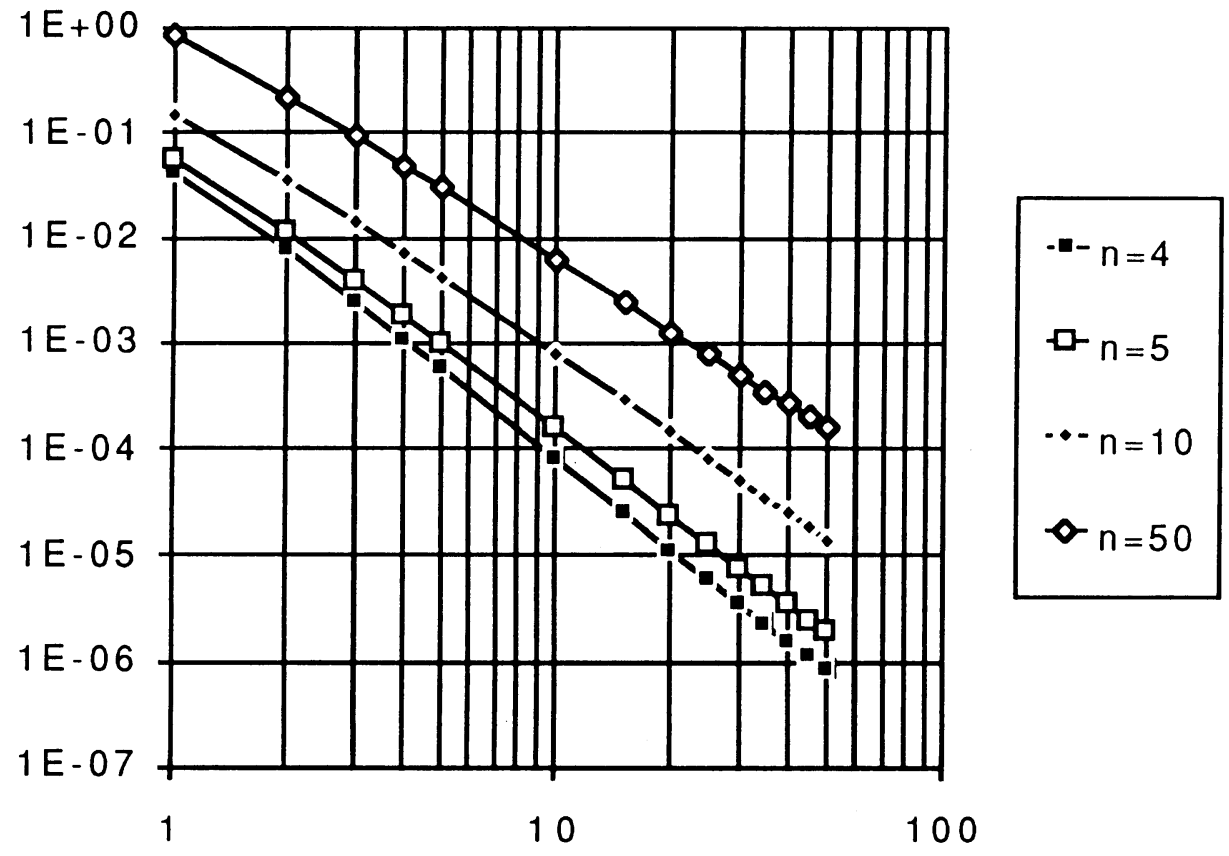

$\Delta \mathrm{n}$

Fig. 1. $f\left(n s^{1} S, m p^{1} P^{0}\right)$ as a function of $\Delta n$, for selected values of $n$ and $m(=n+\Delta n)$.

et al. (1969) estimated the uncertainty of the Coulomb approximation to be about $50 \%$, but they felt that this was quite conservative. Our results support this view; in any case, an uncertainty of $\sim 50 \%$ seems quite acceptable in many astrophysical problems. Further, they may be extrapolated to obtain threshold photoionization cross-sections for magnesium Rydberg states, as shown by Hoang-Binh (1983 and unpublished) for the case of hydrogen. In this connection, it may be noted that an efficient analytical method (also based on the Coulomb approximation), which allows one to deal easily with $n>10$, has been proposed by Hoang-Binh and Van Regemorter (1979).

It is interesting to see how far the $f$-values for transitions between $\mathrm{Mg}$ I Rydberg states deviate from hydrogenic values. Table 2 lists $f$-values for $n f^{1} F^{0}-m g^{1} G$ transitions in $\mathrm{Mg}$ I together with the corresponding hydrogenic values (quantum defects set equal to 0 ). It can be seen that the $f$-values still differ significantly from hydrogenic ones for $n f^{1} F^{0}-m g{ }^{1} G$ transitions, which involve high angular momenta $(l=3,4)$. This should be borne in mind when interpreting solar spectra (Jefferies 1991). Thus, it is only for transitions between $l \geq 4$ Rydberg states that 
TABLE I

Values of $f\left(n p^{1} P^{0}, m s^{1} S\right)$. The last column shows the percentage difference between this work and the results of Moccia and Spizzo (1988).

\begin{tabular}{rrrrr}
\hline$\Delta n$ & $n$ & This Work & MS & Difference(\%) \\
\hline 1 & 4 & $2.97 \mathrm{E}-01$ & $2.99 \mathrm{E}-01$ & -0.53 \\
& 5 & $4.31 \mathrm{E}-01$ & $4.31 \mathrm{E}-01$ & -0.02 \\
& 6 & $5.65 \mathrm{E}-01$ & $5.64 \mathrm{E}-01$ & +0.12 \\
& 7 & $6.98 \mathrm{E}-01$ & $6.97 \mathrm{E}-01$ & +0.24 \\
& 8 & $8.32 \mathrm{E}-01$ & $8.29 \mathrm{E}-01$ & +0.38 \\
2 & 4 & $1.98 \mathrm{E}-02$ & $1.98 \mathrm{E}-02$ & -0.14 \\
& 5 & $2.96 \mathrm{E}-02$ & $2.97 \mathrm{E}-02$ & -0.36 \\
& 6 & $3.89 \mathrm{E}-02$ & $3.91 \mathrm{E}-02$ & -0.57 \\
& 7 & $4.78 \mathrm{E}-02$ & $4.79 \mathrm{E}-02$ & -0.11 \\
3 & 4 & $6.11 \mathrm{E}-03$ & $6.10 \mathrm{E}-03$ & +0.21 \\
& 5 & $9.19 \mathrm{E}-03$ & $9.20 \mathrm{E}-03$ & -0.16 \\
& 6 & $1.20 \mathrm{E}-02$ & $1.20 \mathrm{E}-02$ & +0.33 \\
\hline
\end{tabular}

TABLE II

Values of $f\left(n f^{1} F^{0}, m g^{1} G\right)$

\begin{tabular}{rrrrr}
\hline$\Delta n$ & $n$ & $\mathrm{H}$ & $\mathrm{Mg} \mathrm{I}$ & Difference $(\%)$ \\
\hline 1 & 5 & $1.18 \mathrm{E}+00$ & $1.05 \mathrm{E}+00$ & 13.25 \\
& 10 & $1.08 \mathrm{E}+00$ & $8.19 \mathrm{E}-01$ & 31.31 \\
& 20 & $1.36 \mathrm{E}+00$ & $9.48 \mathrm{E}-01$ & 43.45 \\
& 50 & $2.51 \mathrm{E}+00$ & $1.65 \mathrm{E}+00$ & 51.80 \\
2 & 5 & $2.29 \mathrm{E}-01$ & $2.28 \mathrm{E}-01$ & 0.47 \\
& 10 & $2.72 \mathrm{E}-01$ & $2.34 \mathrm{E}-01$ & 16.25 \\
& 20 & $3.46 \mathrm{E}-01$ & $2.71 \mathrm{E}-01$ & 27.70 \\
& 50 & $6.17 \mathrm{E}-01$ & $4.55 \mathrm{E}-01$ & 35.85 \\
5 & 5 & $2.39 \mathrm{E}-02$ & $2.61 \mathrm{E}-02$ & -8.74 \\
& 10 & $3.99 \mathrm{E}-02$ & $3.78 \mathrm{E}-02$ & 5.38 \\
& 20 & $5.28 \mathrm{E}-02$ & $4.53 \mathrm{E}-02$ & 16.55 \\
& 50 & $8.10 \mathrm{E}-02$ & $7.17 \mathrm{E}-02$ & 25.48 \\
\hline
\end{tabular}


the use of hydrogenic values is fully justified. In this connection, we note that a very efficient method for calculating exact hydrogenic radial integrals, for principal quantum numbers up to 1000 , has been proposed (Hoang-Binh et al. 1979; HoangBinh 1990).

\section{References}

Bashkin, S., Stoner, J.O.: 1975, Atomic Energy Levels and Grotrian Diagrams 1: HydrogenPhosphorus $X V$, North Holland, Amsterdam.

Brault, J., Noyes, R. W.: 1983, Astrophys. J. (Letters) 269, L61.

Chang, E.S., Avrett, E.H., Mauas, P.J., Noyes, R. W., Loeser, R.: 1991,Astrophys. J. (Letters) 379, L79.

Chang, E.S., Noyes, R.W.: 1983, Astrophys. J. (Letters) 275, L11.

Froese-Fisher, C.: $1975 a$, Can. J. Phys. 53, 184.

Froese-Fisher, C.: 1975 b, Can. J. Phys. 53, 338.

Goldberg, L.: 1939, Astrophys. J. 90, 414.

Hoang-Binh, D.: 1983, Astron. Astrophys. 121, L19.

Hoang-Binh, D.: 1990, Astron. Astrophys. 238, 449.

Hoang-Binh, D.: 1991, Astron. Astrophys. 241, L13.

Hoang-Binh, D., Van Regemorter, H.: 1979, J. Phys. B 14, L329.

Hoang-Binh, D., Prud'homme, M., Van Regemorter, H.: 1979, Astrophys. J. (Letters) 230, L127.

Hoang-Binh, D., Brault, P., Picart, J., Tran-Minh, N., Vallee, O.: 1987, Astron. Astrophys. 181, 134.

Jefferies, J.T.: 1991, Astrophys. J. 377, 337.

Moccia, R., Spizzo, P.: 1988, J. Phys. B 21, 1133.

Risberg, G.: 1965, Ark. Fys. 28, 381.

Victor, G.A., Stewart, R. F., Laughlin, C.: 1976, Astrophys. J. Suppl. 31, 237.

Wiese, W.L., Smith, M.W., Miles, B.M.: 1969, Atomic Transitions Probabilities II, Sodium through Calcium, NSRDS-NBS 22, U.S. Government Printing Office, Washington, D.C. 